\title{
Orbital Cellulitis and Secondary Angle Closure: A Rare Presentation of Choroidal Melanoma
}

\author{
Lawrence J. Oh ${ }^{\mathrm{a}, \mathrm{b}}$ Hamish Dunn ${ }^{\mathrm{b}, \mathrm{c}}$ Svetlana Cherepanoffa,b Michel Giblin ${ }^{\mathrm{a}, \mathrm{b}}$ \\ ${ }^{a}$ Save Sight Institute, ${ }^{b}$ University of Sydney, and ${ }^{c}$ Concord Hospital, Sydney, NSW, Australia
}

\section{Established Facts}

- Choroidal melanoma is rarely associated with orbital cellulitis in the literature.

- Orbital cellulitis secondary to choroidal melanoma is particularly sensitive to oral steroids.

\section{Novel Insights}

- Specific imaging characteristics on magnetic resonance imaging may assist in differentiating malignant aetiologies of orbital cellulitis.

- Secondary angle closure may develop from multiple mechanisms in choroidal melanoma.

\section{Keywords}

Choroidal melanoma - Orbital cellulitis · Diagnostic imaging · Secondary cellulitis

\begin{abstract}
We describe a case of choroidal melanoma initially presenting with orbital cellulitis, fulminant conjunctival swelling, and secondary angle closure. Despite treatment with intravenous antibiotics, the patient's condition did not improve. With further investigations including ultrasound scan and magnetic resonance imaging, a high-density lesion was found within the globe. Characteristic imaging findings suggested a malignant origin and the lesion was found to be a melanoma on histopathological analysis.
\end{abstract}

(c) 2017 S. Karger AG, Basel

\section{KARGER}

(C) 2017 S. Karger AG, Basel

E-Mail karger@karger.com

www.karger.com/oop

\section{Clinical Record}

A 63-year-old Caucasian male was referred with presumed orbital cellulitis, after a 1-week history of mild ocular irritation. He was a low hypermetrope, with a history of emphysema, previous alcohol abuse, recurrent tropical skin infections, and post-traumatic stress disorder from work in the Pacific islands. On examination, visual acuity was perception of light in the right eye, and $6 / 5$ in the left with a reverse relative afferent pupillary defect. There was periorbital oedema and erythema, with massive indurated chemosis overlying the cornea limiting full examination (Fig. 1a, b). The pupil was mid-dilated with rubeosis and the anterior chamber was shallow with a 1-mm hyphaema. There was a grey mass visible in the anterior vitreous, but fundal view was impeded by vitritis. Ocular motility was limited in all directions of gaze. Formal intraocular pressure measurement was prevented by the chemosis but the globe was tense to palpation, with resistance to retropulsion.

CT showed orbital cellulitis and high-density material within the globe. US showed a mushroom-shaped lesion emanating from 

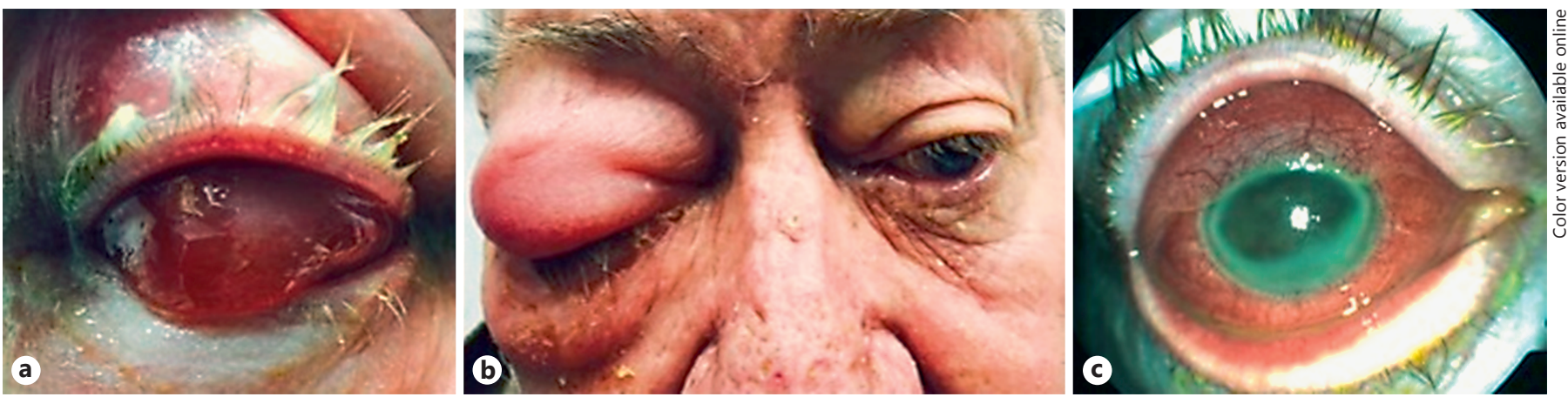

Fig. 1. a, b Periobital oedema and erythema, with massive indurated chemosis overlying the cornea limiting full examination. c Chemosis and orbital inflammation, which has evidently resolved in $\mathbf{b}$ following oral prednisone therapy.

Fig. 2. a MRI image depicting heterogenous intraocular lesion with fluid levels, intermediate-high T1 signal, and low-intermediate T2 signal. b MRI image with restricted diffusion suggestive of high lesion cellularity.
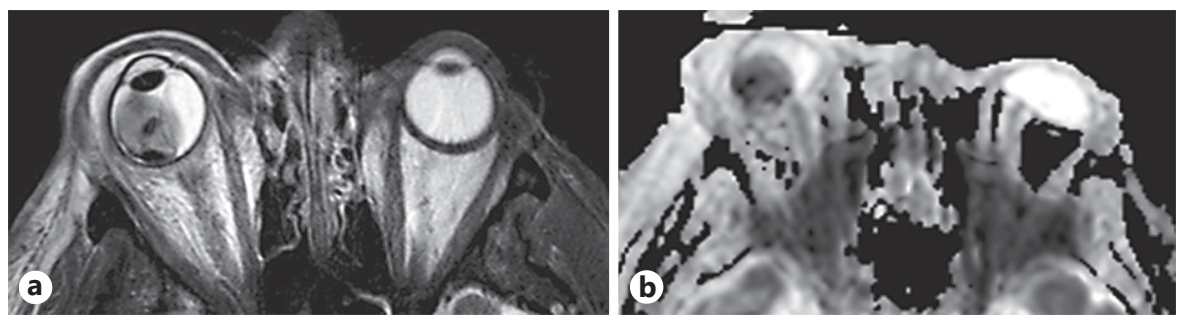

the choroid with low internal reflectivity, and a subtotal retinal detachment.

He was treated with intravenous and topical antibiotics, atropine, and ocular hypotensive agents. Oral prednisone $25 \mathrm{mg}$ was commenced with a rapid resolution of the chemosis and orbital inflammation over 3 days (Fig. 1c). Two days after presentation, a total hyphaema developed and the intraocular pressure was $48 \mathrm{~mm}$ Hg. Subsequent MRI showed a heterogenous intraocular lesion with fluid levels, intermediate-high T1 signal and low-intermediate T2 signal, and restricted diffusion suggestive of high lesion cellularity [1] (Fig. 2a, b).

The differential diagnosis included infective panophthalmitis, given the previous tropical infections. As the MRI and US findings suggested neoplasia rather than an infective ocular lesion, enucleation was performed 1 week post-presentation. Metastatic investigations found no evidence of systemic spread, and no cutaneous lesions were identified.

Histopathological diagnosis was difficult due to extensive tumour necrosis, but specialist referral revealed a choroidal melanoma (pT3NxMx) with clear margins, and evidence of acute on chronic panophthalmitis. The retina was mostly necrotic with long-standing exudative retinal detachment, and there was liquefactive necrosis of the iris and ciliary body. Necrotic tumour in the vitreous had displaced the lens anteriorly and the anterior chamber was filled with exudate. Optic nerve involvement was present with retrolaminar HMB45+ tumour cells, but surgical margins were clear; hence, the patient was monitored without adjuvant chemotherapy or radiotherapy (Fig. 3a, b).

Choroidal Melanoma

\section{Discussion}

Choroidal melanoma is the most prevalent primary ocular malignancy in adults and is usually discovered incidentally on fundoscopy [2]. Presenting symptoms such as floaters or deficiencies in visual acuity or field may be accompanied by ocular inflammation, which can be the first sign of malignant melanoma $[2,3]$.

In rare cases, the tumour can evade detection and eventually result in orbital cellulitis, either via extrascleral extension or inflammation from tumour growth [2]. Inflammatory orbital cellulitis is a rare presentation of choroidal melanoma [2-4]. When a tumour outgrows its blood supply, ischaemia and necrosis can release inflammatory cytokines, resulting in orbital inflammation that is responsive to systemic steroid therapy [2]. Our patient had a characteristic rapid response to steroids.

Ocular hypertension complicates choroidal melanoma in $2 \%$ of cases, usually secondary to iris neovascularisation [5]. Our patient had developed secondary angle closure from multiple mechanisms: necrotic inflammatory change displacing the lens-iris diaphragm anteriorly in a hypermetrope, rubeosis, and hyphaema.

Ocul Oncol Pathol 2018;4:130-133 

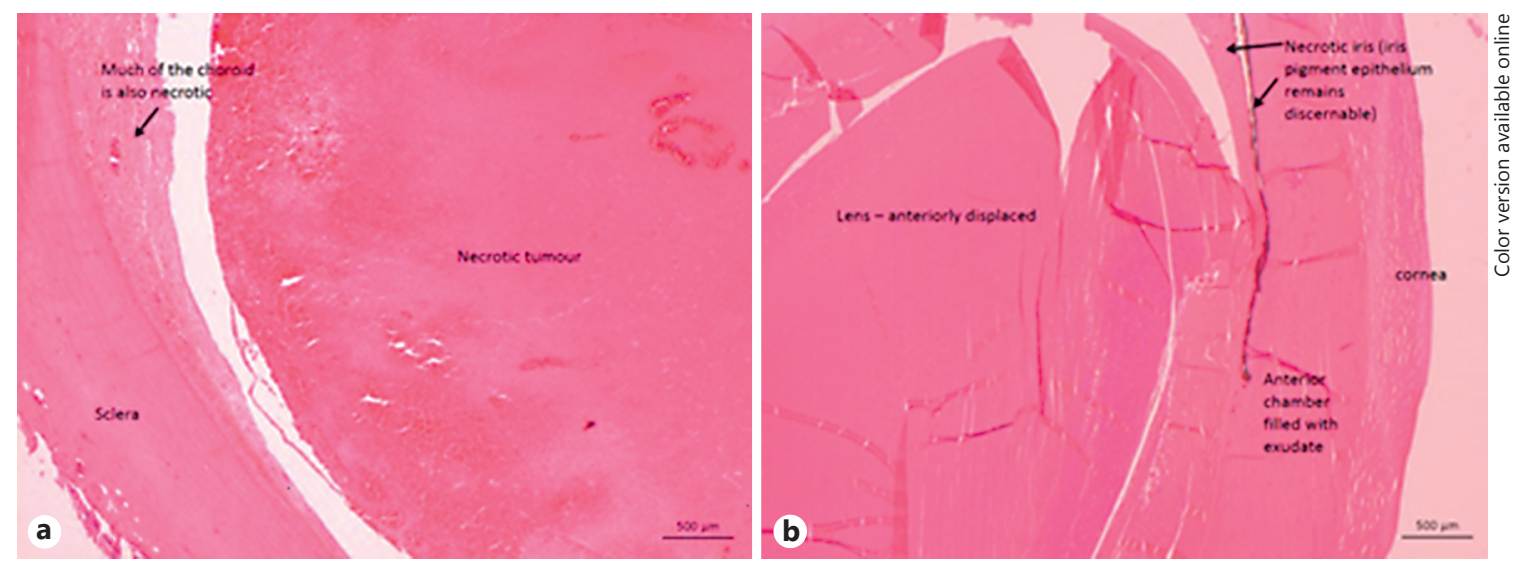

Fig. 3. Haematoxylin and eosin slide showing necrosis of both choroid and tumour within the vitreous cavity (a), which caused anterior displacement of the lens, liquefactive necrosis of the iris, and exudate in the shallowed anterior chamber (b).

Table 1. MRI characteristics of ocular melanoma

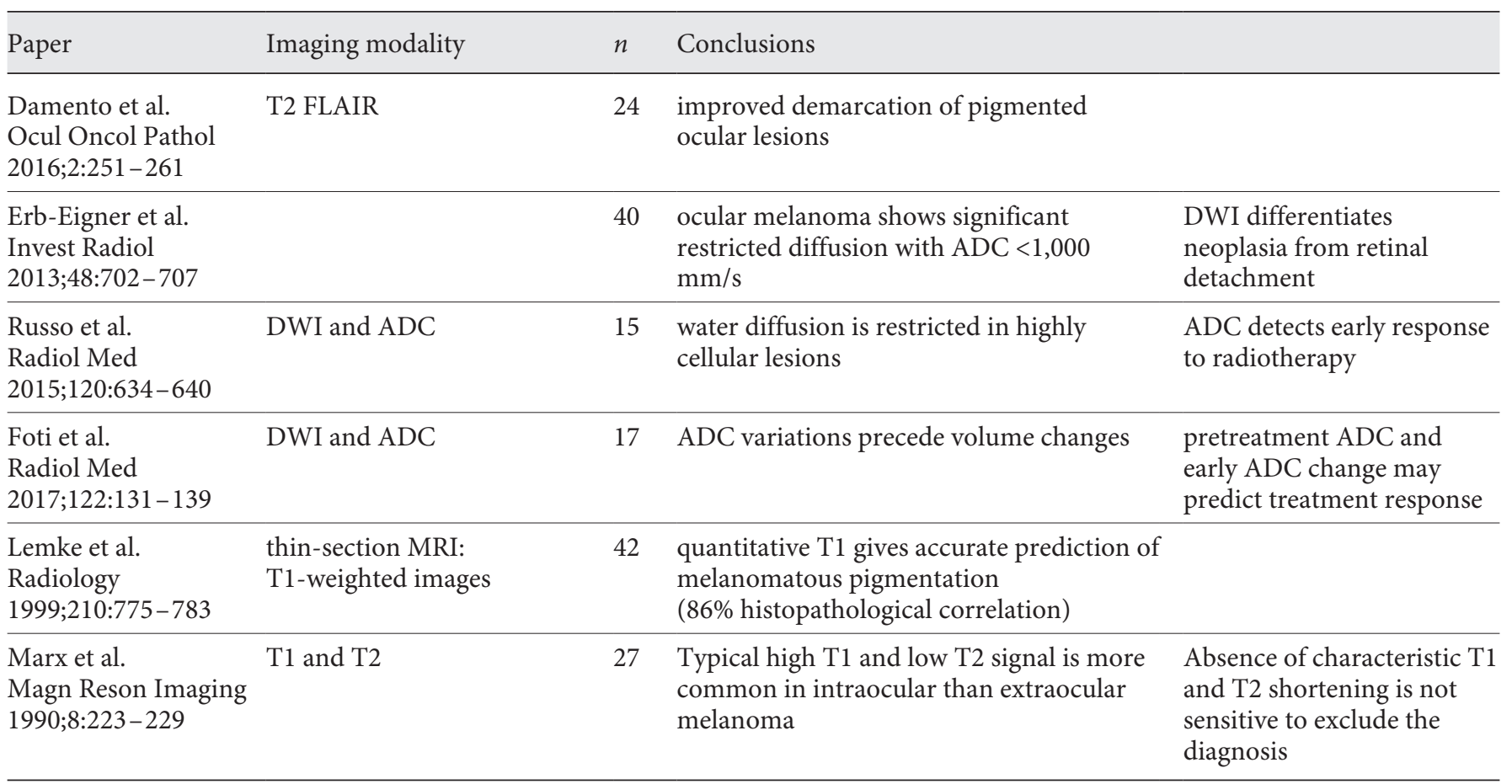

ADC, apparent diffusion coefficient; DWI, diffusion-weighted imaging.

Characteristic imaging features on B-scan ultrasound and magnetic resonance imaging (Table 1) accompanied by histopathologic assessments assist the early diagnosis of choroidal melanomas [2,4].
Our case demonstrates the diagnostic dilemma of choroidal melanoma presenting with secondary orbital cellulitis and secondary angle closure. This highlights the utility of imaging and steroid sensitivity in helping differentiate neoplasia from other causes. 


\section{Acknowledgements}

The authors would like to acknowledge the clinical assistance provided by Dr Raf Ghabrial and Dr Hema Arvind.

\section{Disclosure Statement}

The authors declare no competing/conflicts of interests and there are no financial disclosures.

\section{Statement of Ethics}

Our patient has provided informed consent for publication of this case.

\section{References}

Choroidal Melanoma
1 Marx HF, Colletti PM, Raval JK, Boswell WD, Zee C: Magnetic resonance imaging features in melanoma. Mag Res Imag 1990;8:223-229.

2 Biswas J, Ahuja VK, Shanmugam MP, Kurian R, Fernandez T: Malignant melanoma of the choroid presenting as orbital cellulitis: report of two cases with a review of the literature. Orbit 1999;18:123-130.

3 Fraser DJ, Font RL: Ocular inflammation and haemorrhage as the initial manifestation of uveal malignant melanoma. Incidence and prognosis. Arch Ophthalmol 1979;97:13111314.

4 Nair AG, Kaliki S, Ali MJ, Naik MN, Vemuganti GK: Intraocular malignant melanoma of the choroid presenting as orbital cellulitis. Int Ophthalmol 2014;34:647-650.

5 Shields CL, Shields JA, Shields MB, Augsburger JJ: Prevalence and mechanisms of secondary intraocular pressure elevation in eyes with intraocular tumors. Ophthalmology 1987;94:839-846. 